\title{
Stability analysis of constrained conveyor chain
}

Wang Guojin ${ }^{a}$, Liang Yanshu ${ }^{\text {b,* }}$ and Hong Lin ${ }^{c}$

\author{
School of mechanical engineering, Tianjin Commercial University, Tianmu Town of Beichen District, \\ Tianjin, China \\ a 542971025@qq.com, ${ }^{\mathrm{b}}$ lyshu@tjcu.edu.cn, ${ }^{\mathrm{c}}$ honglinsd@163.com \\ *corresponding author
}

Keywords: Slide constraint, chain drive, polygonal effects, stability

\begin{abstract}
The slideway restrained conveyor chain is a new type of chain drive configuration. Reasonable selection of various parameters is conducive to improving the performance of the chain. In this paper, a special case of the conveyor chain as the research object, calculated its stability and found out the influencing factors. The simulation results show that the polygon effect of the chain can be reduced by reducing the length of the link, increasing the radius of the sprocket and decreasing the speed of the active sprocket. It provides some reference for reducing the polygonal effect of chain drive in chute constrained chain.
\end{abstract}

\section{Introduction}

The polygon effect in the conveyor chain has always been one of the technical problems to be solved. The polygon effect will have a serious impact on the smooth operation of the conveyor chain[1]. Chain drive will bring obvious technical and economic benefits[2][3].Wang Guangjian et[4] designed a new type of high-speed conveyor chain, and through simulation methods, the centroid of the chain plate and the center of mass of the pallet have an impact on the stability of the conveyor chain. Zhang Lingling et[5] verified the theoretical analysis of the angular velocity and unevenness of the driven sprocket in the ordinary chain drive through experimental methods. Yang Renmin et[6] solved the equation by the fourth-order R-K method and obtained the real-time motion state of the driven sprocket. Li Yingjie et[7] analyzed the motion characteristics of the conveyor chain from the perspective of kinematics, and analyzed some factors affecting the polygonal effect of chain transmission. Li Shujing et[8] obtained the compensation trajectory of the free pin in the large pitch conveyor chain from both statics and dynamics. In this paper, through a kind of chute-constrained conveyor chain model[9], the displacement difference of the main driven sprocket is calculated, and some factors affecting the displacement difference are found out.

\section{Analysis and Calculation}

The chain center line of the traditional chain drive conveyor chain and the sprocket indexing circle alternately have the phenomenon of tangency and phase cutting during the movement, which leads to the continuous change of the chain speed and acceleration, and the movement of the chain when the chain continues to appear. It is the polygon effect that exists in ordinary chain drives.

\subsection{Slide Constrained Conveyor Chain Configuration}

This paper establishes a transport chain model that constrains the links of the chain to a fixed orbit, as shown in Figure 2. The right driving sprocket rotates clockwise at an angular velocity $\omega_{1}=0.5 \mathrm{rad} / \mathrm{s}$. The length of each pin is $\mathrm{p}=262$, the center of the left and right sprocket is $\mathrm{O}_{2} 、 \mathrm{O}_{1}$, and the radius are $r=185$, and the distance between them is $\mathrm{D}$. Thus, the sprocket can accommodate three pins at the same time, and the number of teeth of the sprocket is four. The pin shafts are numbered P1-P12 clockwise from the beginning to the position of the drive sprocket, which is known to be $\angle \mathrm{P}_{1} \mathrm{O}_{1} \mathrm{P}_{2}=90^{\circ}$. Since the chain has a periodicity during the movement, from the pin $\mathrm{P}_{1}$ movement to the pin $\mathrm{P}_{2}$, it is possible to analyze the motion of one cycle. $\alpha_{1}$ is the angle between 
$\mathrm{P}_{1} \mathrm{O}_{1}$ and positive direction of the $\mathrm{y}$-axis and $\alpha_{2}$ is the angle between $\mathrm{P}_{2} \mathrm{O}_{1}$ and positive direction of the $\mathrm{x}$-axis. $\alpha_{7}$ is the angle between $\mathrm{P}_{7} \mathrm{O}_{2}$ and the negative direction of the $\mathrm{y}$-axis and $\alpha_{8}$ is the angle between $\mathrm{P}_{8} \mathrm{O}_{2}$ and negative direction of the X-axis.In this model, $\alpha_{1}$ equals $\alpha_{2}$ and $\alpha_{7}$ equals $\alpha_{8}$.

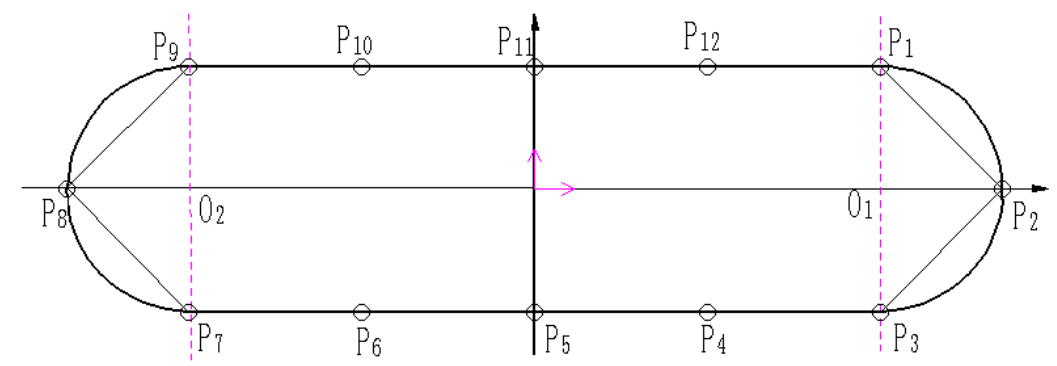

Figure 1 Trajectory constrained chain drive pin position

\subsection{Polygon Effect Representation}

During the movement, the pin speeds at the left and right sprocket positions vary greatly, which is the polygonal effect of the chain. In order to express the strength of the polygon effect, the displacement changes of the pin shafts of the left and right sprocket in one cycle are calculated respectively. The larger the displacement difference is, the more obvious the polygon effect is, and the more unstable the conveyor chain is. Here is the calculation process.

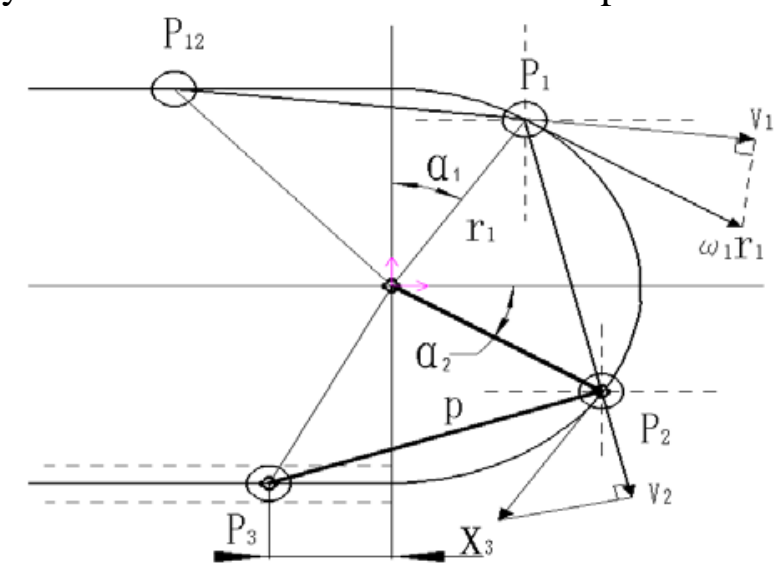

Figure 2 Speed analysis of right chain wheel

From the motion analysis diagram, the pin $\mathrm{P}_{1}$ is on the upper semicircle of the drive sprocket, its speed

$$
v_{1}=\omega_{1} \mathrm{r}_{1} \cos \left[\alpha_{1}-\arcsin \frac{\mathrm{r}-\mathrm{r} \cos \alpha_{1}}{p}\right] \quad \alpha_{1} \in\left[0, \frac{\pi}{2}\right]
$$

When calculating the pin, the model can be converted into a crank slider mechanism. The pin is on the straight slide, and the distance from the lower cut point is recorded as shown in Figure 2.

$$
x_{3}=\sqrt{p^{2}-\left(r_{1}-r_{1} \sin \alpha_{2}\right)^{2}}-r_{1} \cos \alpha_{2}=\left(\sqrt{\frac{\cos ^{2} \alpha_{2}}{2}+\sin \alpha_{2}}-\frac{\sqrt{2}}{2} \cos \alpha_{2}\right) p
$$

The speeds $\mathrm{v}_{4}, \mathrm{v}_{5}$, and $\mathrm{v}_{6}$ of the pins $\mathrm{P}_{4}, \mathrm{P}_{5}$, and $\mathrm{P}_{6}$ are equal to the speed $\mathrm{v}_{3}$ of the pin $\mathrm{P}_{3}$, and the distance from the pin $\mathrm{P}_{6}$ to the lower left point is $\mathrm{X}_{6}$, so

$$
x 6=D-3 p-x 3
$$

As shown in Figure 3, in the triangle $\mathrm{P}_{6} \mathrm{O}_{2} \mathrm{P}_{7}$

$$
\alpha_{7}=\arccos \frac{r^{2}+r^{2}+x_{6}^{2}-p^{2}}{2 r_{2} \sqrt{r_{2}^{2}+x_{6}^{2}}}-\arctan \frac{x_{6}}{r_{2}}
$$




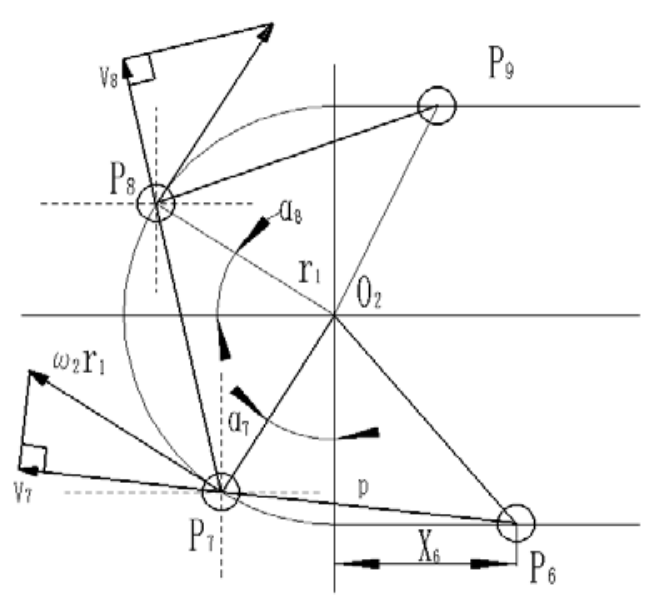

Figure 3 Left sprocket speed analysis

Pin $\mathrm{P}_{8}$ on the upper half of the left sprocket, so

$$
v_{8}=\omega_{2} \mathrm{r}_{2} \cos \left[\alpha_{8}+\arcsin \frac{\mathrm{r}_{2} \cos \alpha_{8}-\mathrm{r}_{2} \sin \alpha_{7}}{p}\right]
$$

Based on the equations (1) and (9), the displacement difference can be further solved.

$$
\begin{array}{r}
s_{1}-s_{8}=\int v_{1} d t-\int v_{8} d t==\sum_{t=0}^{n} \omega_{1} r_{1} \cos \left[\omega_{1} t-\arcsin \frac{r-r \cos \left(\alpha_{1}^{\prime}+\omega_{1} t\right)}{p}\right] t- \\
\sum_{t=0}^{n} \omega_{2} r_{2} \cos \left[\omega_{2} t+\arcsin \frac{r_{2} \cos \omega_{2} t-r_{2} \sin \left(\frac{\pi}{2}+2 \arcsin \frac{p}{2 r}+\omega_{2} t\right)}{p}\right] t \quad\left(n \in\left(0,2 \arcsin \frac{p}{2 r}\right)\right)
\end{array}
$$

\subsection{The Influence of Different Factors on the Stability of the Conveyor Chain}

It can be seen from the analysis that the factors affecting the polygon effect are $r$, p, etc., and the effects of the polygon effect are analyzed one by one below. Firstly, the influence of the link p on the polygon effect is analyzed. For the convenience of calculation, it is assumed that the angular velocities of the left and right sprocket are equal. It is assumed that after changing the link size p, the initial position of the pin is always at the upper right tangent point, and the initial position of the pin is at the left chain. At the midpoint of the wheel, the motion in a period [0, 2arcsin(p/2r)] is analyzed, and all conditions except the center distance are equal. The displacement difference in the case where the link p is 222, 242, 262, 282, and 302 using matlab is as shown in FIG. 5. It can be seen from the figure that the larger the link $\mathrm{p}$ is, the larger the displacement difference is, that is, the more obvious the polygon effect is. Under the same conditions, the analysis of $\omega$ is $0.3,0.4,0.5,0.6$, 0.7 , respectively. It can be seen from the figure that the larger the angular velocity, the more obvious the polygonal effect.

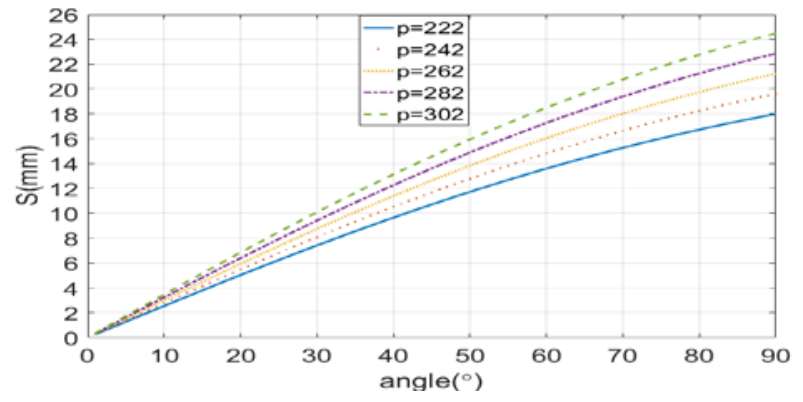

Figure 4 Effect of link length p on displacement difference 


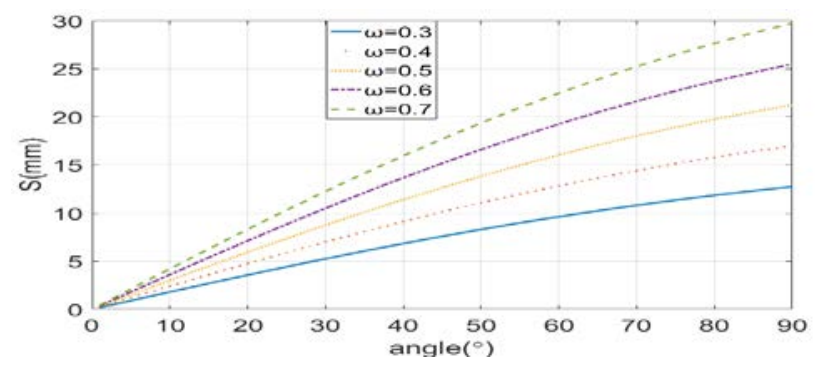

Figure 5 Effect of angular velocity on displacement difference

\section{Conclusion}

Through the analysis of the motion of the glide-constrained conveyor chain in special cases, a calculation method for measuring stability is obtained, and the stability is related to factors such as $\mathrm{r}$ and p. Under suitable conditions, the chain length can be reduced or the sprocket radius can be increased, and the driving sprocket rotation speed can be reduced to reduce the polygon effect in the chain transmission, thereby improving the stability of the conveyor chain during the movement.

\section{Acknowledgements}

The work in this paper is supported by Tianjin natural science foundation under the Contract No. 17JCZDJC38200. The authors wish to acknowledge the help of fellow, teachers and researchers in Tianjin University of Commerce who made the work possible.

\section{references}

[1] Liang Zhimin, An Zongwen, Li Zhaohui. Analysis of the stability of conveyor chain operation [J]. Lifting and transporting machinery, 2003 (02): 18-20.

[2] Meng Fanzhong, Ye Bin. Chain Drive Technical Manual [M]. Beijing: Mechanical Industry Press, 2016: 1 - 2.

[3] Meng Fanzhong.The Development History and Technology Research Status and Prospect of China's Chain Transmission Industry[J].Journal of Mechanical Transmission,2016,40(11):1-7.

[4]Wang Guangjian, Fan Yumeng. Design, Simulation and Experiment of High Speed Conveyor Chain Transmission[J]. Journal of Mechanical Engineering, 2013, 49(13): 158-163.

[5]ZHANG Lingling, LU Tianxi, WU Luming, ZHANG Xianwen.Experimental Study on the Polygonal Effect of Chain Transmission[J].Mechanical Engineering \& Automation,2010(04):97-99.

[6]YANG Renmin, ZHANG Xuechang, HAN Junxiang, LI Jialin. Construction and Simulation of Real-time Transmission Mathematical Model of Chain Drive Polygon Effect[J].Machinery Science and Technology,2017,36(06):821-826.

[7] Li Yingjie, Hong Lin. Stability analysis of conveyor chain operation [J]. Lifting and transporting machinery, 2014 (07): 77-79.

[8] Li Shujing, Hong Lin, Liang Yanshu. Trajectory compensation of a large pitch constrained conveyor chain[J]. Mechanical Transmission, 2017, 41(02): 131-133+142.

[9] Li Xiao, Hong Lin, Li Desheng. A Compensation Method for Polygonal Effect of Chain Transmission[J].Journal of Tianjin University of Technology,2008,24(1):11-13. 